\section{Diferenciais de mortalidade entre indígenas e não indígenas no Brasil com base no Censo Demográfico de 2010}

\author{
Differences in mortality between indigenous and \\ non-indigenous persons in Brazil based on the \\ 2010 Population Census
}

\section{Diferenciales de mortalidad entre indígenas y no indígenas en Brasil, en base al Censo Demográfico de 2010}

COMUNICAÇÃO BREVE

BRIEF COMMUNICATION

\author{
Marden Barbosa de Campos 1 \\ Gabriel Mendes Borges 2 \\ Bernardo Lanza Queiroz ${ }^{3}$ \\ Ricardo Ventura Santos 4,5
}

doi: 10.1590/0102-311X00015017

\section{Resumo}

Estimativas sobre os diferenciais de mortalidade adulta ou geral para a população indígena no Brasil são inexistentes, embora a construção destes indicadores seja de extrema importância para a redução das iniquidades sociais em termos de saúde verificada para este segmento populacional. Nas últimas décadas, avanços significativos ocorreram no Brasil na direção de reverter a falta de dados referentes aos indígenas nas estatísticas nacionais. O objeto desta comunicação é apresentar estimativas de mortalidade para indígenas e não indígenas em diferentes grupos de idades, baseadas nos dados do Censo Demográfico de 2010. Isso é feito com base no quesito de óbitos investigado em domicílios particulares. Os resultados encontrados indicam diferenças expressivas nas probabilidades de morte entre indígenas e não indígenas em todos os grupos de idades selecionados, para ambos os sexos. Observa-se que esses diferenciais são mais pronunciados na infância, principalmente para o sexo feminino. Os indicadores elaborados corroboram o fato de que os povos indígenas do Brasil encontram-se em uma situação de extrema vulnerabilidade em temos de saúde, estimando, de forma inédita, a magnitude destes diferenciais.

Mortalidade; Censos; População Indígena

\section{Correspondência}

M. B. Campos

Rua Desembargador Alarico Barroso 385, apto. 204, Belo Horizonte, MG 31310-380, Brasil.

mardencampos@gmail.com

1 Departamento de Sociologia, Universidade Federal de Minas Gerais, Belo Horizonte, Brasil.

2 Instituto Brasileiro de Geografia e Estatística, Rio de Janeiro, Brasil.

3 Centro de Desenvolvimento e Planejamento Regional,

Universidade Federal de Minas Gerais, Belo Horizonte, Brasil. 4 Escola Nacional de Saúde Pública Sergio Arouca, Fundação Oswaldo Cruz, Rio de Janeiro, Brasil.

5 Museu Nacional, Universidade Federal do Rio de Janeiro, Rio de Janeiro, Brasil. 
A redução das iniquidades no campo da saúde no Brasil demanda, necessariamente, estratégias de mensuração e construção de indicadores de saúde para grupos populacionais específicos. Não há, entretanto, estudos recentes e representativos sobre os níveis de mortalidade adulta ou geral para a população indígena no Brasil, prevalecendo investigações acerca da mortalidade infantil 1,2. Tampouco há estimativas de esperança de vida ao nascer para os indígenas, dimensão que tem sido explorada recentemente em análises internacionais 3 . No que diz respeito aos debates sobre desigualdades étnico-raciais no Brasil, ainda estão por ser geradas tábuas de vida e estimativas de mortalidade geral segundo cor ou raça da população.

Nas últimas décadas, avanços significativos ocorreram no Brasil na direção de reverter a falta de dados referentes às populações indígenas nas estatísticas nacionais 4,5,6. Isso se aplica aos censos demográficos nacionais que, desde 1991, têm gerado dados sobre a categoria "indígena" no quesito sobre cor ou raça $6,7,8$.

O objeto desta comunicação é apresentar estimativas de mortalidade para indígenas e não indígenas em diferentes grupos de idades, baseadas nos dados de óbitos nos domicílios coletados pelo Censo Demográfico de 2010.

\section{Fonte de dados e métodos}

A estimação de níveis e padrões de mortalidade no Brasil é dificultada por problemas de cobertura, principalmente pela subdeclaração de óbitos e por erros de declaração de idade 9,10 . Isso ocorre tanto nos dados de censos e outras pesquisas sóciodemográficas, como nos registros administrativos do Sistema de Informações sobre Mortalidade (SIM) do Ministério da Saúde e das estatísticas do Registro Civil. Para lidar com esses problemas, têm sido empregados métodos demográficos indiretos de estimação de mortalidade, que muitas vezes envolvem a necessidade de compatibilizar dados oriundos de fontes diferentes 11 .

O Censo Demográfico de 2010 oferece uma oportunidade ímpar de se realizar estimativas de mortalidade com base em apenas uma fonte (óbitos e população total), embora sejam necessários ajustes nos dados de óbito declarados. Especificamente, no último Censo foi perguntado quanto à ocorrência de óbitos entre pessoas que moravam com o entrevistado, incluindo sexo e idade do falecido, nos 12 meses anteriores à data de referência do Censo (31 de julho de 2010).

Uma investigação recente avaliou a qualidade da informação sobre óbitos no Censo de 2010, considerando-a satisfatória para a realização de estimativas de mortalidade 12 . Contudo, embora não inviabilize a sua utilização, a informação apresenta alguns problemas, como uma possível confusão sobre como reportar óbitos nos casos de dissolução dos domicílios após o falecimento de um dos moradores. Outro problema foi a impossibilidade de encontrar informantes para reportar o óbito em situações específicas, o que se mostrou particularmente relevante devido à elevada proporção de domicílios unipessoais, principalmente entre a população idosa.

No Censo de 2010, o quesito de cor ou raça foi aplicado ao universo da população (questionários básico e da amostra). Isso confere a possibilidade de utilizá-lo de forma desagregada tanto em termos espaciais como em relação a atributos individuais, sem limitações decorrentes do tamanho amostral. Esse aspecto é particularmente relevante, dado que a população indígena (818 mil indivíduos) representa menos de $0,5 \%$ do total da população brasileira 6 .

O quesito de óbitos foi investigado somente em domicílios particulares. Nesse sentido, de forma a manter consistência entre numerador e denominador no cálculo das taxas de mortalidade, a população residente em domicílios coletivos não foi considerada no presente estudo. Como essa população equivale a apenas $0,3 \%$ da população do Brasil, esse procedimento não gera impacto significativo nos resultados.

Em relação à estimativa de mortalidade por cor ou raça com base em dados censitários, é importante enfatizar que não há informação sobre a cor ou a raça do indivíduo que faleceu 1, sendo necessário aplicar um procedimento metodológico adicional para realizar a estimativa. Na busca por uma aproximação para a cor ou a raça do indivíduo falecido, diferentes alternativas foram testadas no âmbito desta investigação: (1) domicílios com pelo menos 1 morador indígena; (2) domicílios com mais da metade dos moradores indígenas; (3) todos os membros do domicílio são indígenas; (4) 
responsável pelo domicílio classificado como indígena. Como as estimativas de mortalidade foram semelhantes entre todas as alternativas (resultados não apresentados), as análises foram detalhadas com base na estratégia (4), qual seja: atribuir ao óbito a cor ou a raça do responsável pelo domicílio (indígena ou não indígena).

Taxas específicas de mortalidade, dadas pela razão entre número de óbitos reportados e a população total em cada grupo de idades, foram calculadas para três intervalos de idades baseando-se nos dados do universo do Censo: (a) menores de 5 anos; (b) entre 5 e 14 anos; (c) entre 15 e 59 anos. Com base nessas estimativas, foram calculadas as probabilidades de morte entre duas idades exatas, $x$ e $x+n\left({ }_{n} q_{x}\right)$. Desse modo, obtiveram-se as probabilidades de morte $\left({ }_{5} q_{0},{ }_{10} q_{5},{ }_{45} q_{15}\right)$ para indígenas e não indígenas de diferentes grupos de idades e para homens e mulheres, separadamente. Sendo a probabilidade de morte fortemente relacionada ao sexo e à idade, essa estratégia permite avaliar um amplo espectro do fenômeno. Não foram realizadas estimativas isoladas para aqueles com 60 anos ou mais, dadas as limitações da informação para este estrato da população, conforme mencionado. Os níveis da mortalidade para indígenas e não indígenas foram ajustados pelos fatores propostos para o Brasil por Queiroz \& Sawyer 12, de 1,23 para mulheres e 1,17 para homens.

\section{Resultados}

No Censo de 2010 foram reportados 1.034.418 óbitos (4.740 indígenas e 1.029 .678 não indígenas) em 1.014.508 domicílios (4.628 indígenas e 1.009 .880 não indígenas). Uma proporção mais elevada de domicílios classificados como indígenas apresentou óbitos $(2,19 \%)$, se comparada aos não indígenas $(1,77 \%)$.

Há diferenças expressivas nas probabilidades de morte entre indígenas e não indígenas em todos os grupos de idades, e de forma mais pronunciada para o sexo feminino (Tabela 1 e Figura 1). As diferenças entre indígenas e não indígenas têm maior expressão na infância, entre 0 e 4 anos de idade, em que as taxas são duas vezes maiores do que entre os não indígenas. A diferença também é acentuada entre os jovens com até 14 anos, embora ligeiramente menor do que na infância. Em relação aos adultos, a mortalidade indígena continua superior àquela dos não indígenas, embora em menor magnitude: $28 \%$ maior para os homens e $19 \%$ para as mulheres.

A Figura 1 mostra as diferenças das taxas por idade, ilustrando os números apresentados pelos indicadores calculados. Observa-se a persistência de níveis mais altos de mortalidade entre os indígenas ao longo do ciclo de vida analisado, principalmente para as mulheres, embora a diferença diminua à medida que a idade avança.

\section{Comentários finais}

Investigação recente realizada em aproximadamente duas dezenas de países registrou que os indígenas tendem a apresentar indicadores de saúde e socioeconômicos menos favoráveis do que os não indígenas ${ }^{3}$. Embora dados referentes ao Brasil tenham sido considerados no estudo, no que diz respeito à mortalidade, eles se restringiram ao componente infantil. $O$ presente trabalho é um primeiro

Tabela 1

Taxas específicas de mortalidade por sexo e grupos de idades selecionados para indígenas e não indígenas, em mil habitantes. Brasil, 2010.

\begin{tabular}{|c|c|c|c|c|}
\hline Grupo de idades & Mulheres indígenas & Mulheres não indígenas & Homens indígenas & Homens não indígenas \\
\hline${ }_{5} q_{0}(\% 0)$ & 34,6 & 17,4 & 38,0 & 19,9 \\
\hline${ }_{10} q_{5}(\% 0)$ & 7,0 & 3,7 & 8,3 & 4,7 \\
\hline${ }_{45} q_{15}(\% 0)$ & 147,0 & 114,8 & 255,1 & 214,7 \\
\hline
\end{tabular}

Fonte: Instituto Brasileiro de Geografia e Estatística 6. 
Figura 1

Taxa específica de mortalidade por sexo, indígenas e não indígenas. Brasil, 2010.

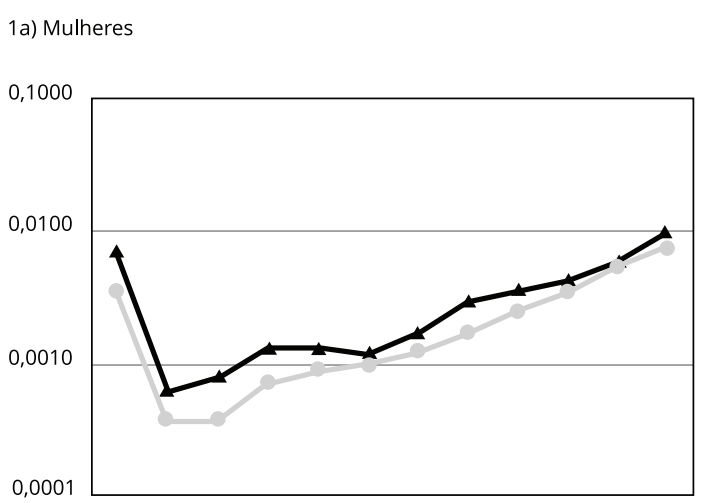

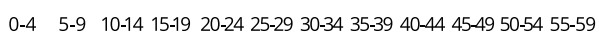

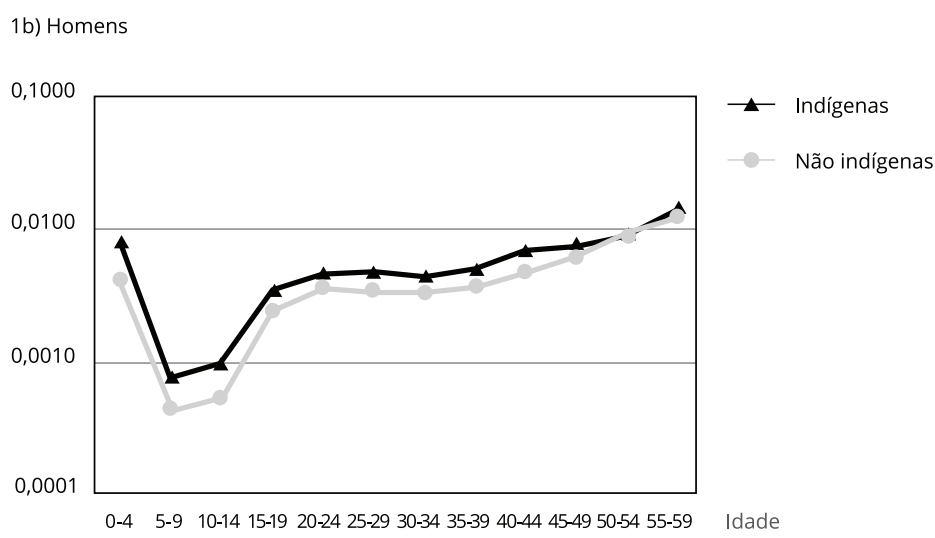

Fonte: Instituto Brasileiro de Geografia e Estatística 6.

esforço de apresentar estimativas de mortalidade no Brasil para indígenas de diferentes idades. Os resultados apontam para diferenças marcantes, com níveis de mortalidade muito mais elevados para os indígenas em todos os grupos de idades considerados, para ambos os sexos. Esses achados estão alinhados a diversas outras análises que apontam para as marcantes desigualdades em saúde dos indígenas no Brasil 3,4,5.

As condições de saúde dos povos indígenas têm sido tema de crescente interesse na América Latina, especialmente no que diz respeito aos esforços para reduzir as disparidades em relação a outros segmentos populacionais 4,13. A disponibilização de indicadores de saúde representativos e confiáveis para os indígenas é um importante aspecto para se alcançar esses objetivos 3 .

Até o presente, os dados censitários, que constituem uma das fontes mais representativas em escala nacional, têm sido pouco explorados sob essa vertente. As análises aqui apresentadas apontam para o potencial de uso do Censo de 2010 para a investigação da mortalidade de indígenas, assim como de não indígenas, no Brasil. 


\section{Colaboradores}

M. B. Campos contribuiu na concepção geral, elaboração do texto, gráficos e tabelas, revisão final e submissão. G. M. Borges colaborou na elaboração dos indicadores, extração dos dados, elaboração de gráficos, tabelas e revisão final da versão para publicação. B. L. Queiroz colaborou na discussão dos indicadores e dos fatores de ajuste, inclusão de referências e revisão final. R. V. Santos contribuiu na concepção geral, redação e revisão final.

\section{Agradecimentos}

À Fundação Carlos Chagas Filho de Amparo à Pesquisa do Estado do Rio de Janeiro (Faperj, E-26/102.352/2013, Bolsa "Cientista de Nosso Estado"), à Fundação de Amparo à Pesquisa do Estado de Minas Gerais (FAPEMIG, Processo APQ01053-16) e à Coordenação de Aperfeiçoamento de Pessoal de Nível Superior (Capes).

\section{Referências}

1. Caldas A, Santos RV, Borges GM, Valente JG, Portela MC, Marinho GL. Mortalidade infantil segundo cor ou raça com base no Censo Demográfico de 2010 e nos Sistemas Nacionais de Informação em Saúde. Cad Saúde Pública; no prelo.

2. Wong LLR, Barros JS, Santos W. Mortalidad infantil e infantojuvenil en Brasil según sexo y color de la piel. In: Wong LLR, Sánchez JA, coordinador. Situación de la población afrodescendiente e indígena en América Latina. Belo Horizonte: Asociación Latinoamericana de Población; 2014. p. 79-98. (Série e-Investigaciones, 4).

3. Anderson IA, Robson B, Connolly M, Al-Yaman F, Bjertness E, King A, et al. Indigenous and tribal peoples' health (The Lancet-Lowitja Institute Global Collaboration): a population study. Lancet 2016; 388:131-57.

4. Cardoso AM, Santos RV, Coimbra Jr. CEA, Garnelo L, Chaves MBG. Políticas públicas de saúde para os povos indígenas. In: Giovanella L, Escorel S, Lobato LVS, Noronha JC, Carvalho AI, organizadores. Políticas e sistemas de saúde no Brasil. 2a Ed. Rio de Janeiro: Editora Fiocruz/Centro Brasileiro de Estudos de Saúde; 2012. p. 911-32.

5. Coimbra Jr. CEA, Santos RV, Welch JR, Cardoso AM, Souza MC, Garnelo L, et al. The First National Survey of Indigenous People's Health and Nutrition in Brazil: rationale, methodology, and overview of results. BMC Public Health 2013; 13:52.
6. Instituto Brasileiro de Geografia e Estatística. Censo Demográfico de 2010. Características gerais dos indígenas. Resultados do universo. Rio de Janeiro: Instituto Brasileiro de Geografia e Estatística; 2012.

7. Campos MB, Estanislau BT. Demografia dos povos indígenas: os Censos Demográficos como ponto de vista. Rev Bras Estud Popul 2016; 33:441-9.

8. Pereira NOM. Avanços na captação de dados sobre a população indígena no Censo Demográfico 2010. Rev Bras Estud Popul 2016; 33:423-30.

9. França E, de Abreu DX, Rao C, Lopez AD. Evaluation of cause-of-death statistics for Brazil, 2002-2004. Int J Epidemiol 2008; 37:891-901.

10. Lima EEC, Queiroz, BL. Evolution of the deaths registry system in Brazil: associations with changes in the mortality profile, under-registration of death counts, and ill-defined causes of death. Cad Saúde Pública 2014; 30:1721-30.

11. Paes NA. Avaliação da cobertura dos registros de óbitos dos estados brasileiros em 2000. Rev Saúde Pública 2005; 39:882-90.

12. Queiroz BL, Sawyer DOT. O que os dados de mortalidade do Censo de 2010 podem nos dizer? Rev Bras Estud Popul 2012; 29:225-38.

13. Del Popolo F, Cunha EMGP, Ribotta B, Azevedo $\mathrm{M}$, organizadores. Pueblos indígenas y afrodescendientes en América Latina: dinámicas poblacionales diversas y desafios comunes. Rio de Janeiro: Asociación Latinoamericana de Población; 2011. 


\section{Abstract}

There have been no previous estimates on differences in adult or overall mortality in indigenous peoples in Brazil, although such indicators are extremely important for reducing social iniquities in health in this population segment. Brazil has made significant strides in recent decades to fill the gaps in data on indigenous peoples in the national statistics. The aim of this paper is to present estimated mortality rates for indigenous and nonindigenous persons in different age groups, based on data from the 2010 Population Census. The estimates used the question on deaths from specific household surveys. The results indicate important differences in mortality rates between indigenous and non-indigenous persons in all the selected age groups and in both sexes. These differences are more pronounced in childhood, especially in girls. The indicators corroborate the fact that indigenous peoples in Brazil are in a situation of extreme vulnerability in terms of their health, based on these unprecedented estimates of the size of these differences.

Mortality; Censuses; Indigenous Population

\section{Resumen}

Las estimativas sobre los diferenciales de mortalidad adulta o general en los pueblos indígenas de Brasil son inexistentes, pese a que la construcción de estos indicadores sea de extrema importancia para la reducción de las inequidades sociales, en términos de salud verificada para este segmento poblacional. En las últimas décadas, se produjeron avances significativos en Brasil, con el fin de revertir la falta de datos referentes a las poblaciones indígenas en las estadísticas nacionales. El objeto de esta comunicación es presentar estimativas de mortalidad para indígenas y no indígenas en diferentes grupos de edades, basadas en los datos del Censo Demográfico de 2010. Esto se realiza basándonos en el apartado de óbitos investigado en domicilios particulares. Los resultados hallados indican diferencias expresivas en las probabilidades de muerte entre indígenas y no indígenas, en todos los grupos de edades seleccionados, para ambos sexos. Se observa que esos diferenciales son más pronunciados en la infancia, principalmente para el sexo femenino. Los indicadores elaborados corroboran el hecho de que los pueblos indígenas de Brasil se encuentran en una situación de extrema vulnerabilidad en términos de salud, estimando, de forma inédita, la magnitud de estos diferenciales.

Mortalidad; Censos; Población Indígena

Recebido em 31/Jan/2017

Versão final reapresentada em 06/Abr/2017

Aprovado em 25/Abr/2017 